\title{
Prevalencia de lesiones por sobreuso en jugadores de las selecciones de rugby de Chile y Uruguay
}

\section{Overuse Injury Prevalence in National Team Rugby Players of Chile and Uruguay}

\author{
Michael Marsalli ${ }^{1}$ Marcelo Santurio ${ }^{2} \quad$ Cristián Garrido $^{3}$ Oscar Sepúlveda ${ }^{4}$ \\ ${ }^{1}$ Traumatólogo, Federación Chilena de Rugby; Equipo de Hombro \\ Hospital del Trabajador; Equipo Traumatología Clínica Universidad \\ de los Andes, Santiago, Chile \\ 2 Médico, Unión Uruguaya de Rugby, Montevideo, Uruguay \\ ${ }^{3}$ Becario Medicina de Urgencias, Universidad de los Andes, Santiago, \\ Chile \\ ${ }^{4}$ Médico Cirujano, Santiago, Chile \\ Rev Chil Ortop Traumatol 2017;58:2-6.

\begin{abstract}
Address for correspondence Michael Marsalli San Martín, MD, Federación Chilena de Rugby, Marcel Duhaut 2949, Providencia, Santiago, Chile (e-mail: dr.marsalli@deportedoc.com).
\end{abstract}

\section{Resumen}

\section{Palabras clave}

- rugby

- lesiones por sobreuso

- lesiones en rugby

\section{Abstract}

Objetivo Determinar la prevalencia de lesiones por sobreuso en las selecciones de rugby adulta y juvenil de Chile y Uruguay. Objetivos secundarios: 1 . Describir el impacto en el rendimiento, factores de riesgo y las diferencias entre adultos y juveniles; 2 . Evaluar la definición de lesión según tiempo perdido para el estudio de las lesiones por sobreuso.

Metodología Grupo de estudio: selecciones adultas y juveniles de rugby de Chile y Uruguay (106 jugadores). Se utilizó el Oslo Sports Trauma Research Centre Overuse Injury Questionnaire, para el registro y la evaluación de las lesiones. Se definió lesión por sobreuso como todos los problemas articulares de origen no traumático, ocurridos hasta los 7 días previos al registro. Se registró la edad, peso, talla, posición de juego y volumen de entrenamiento para el análisis de factores de riesgo.

Resultados La prevalencia fue de un $31 \%$. Solo el $6 \%$ requirió más de 24 horas de reposo deportivo. El principal factor de riesgo fue la edad (OR 1,12 IC 1,017-1,24), aumentando un $12 \%$ por cada año cumplido. Se observó una mayor prevalencia en los adultos y los juveniles presentaron un puntaje mayor de gravedad (23,2 versus $15,8 p=0,03)$.

Conclusiones La prevalencia de lesiones por sobreuso en este grupo fue alta. El impacto en el rendimiento en la mayoría fue leve, siendo mayor en los juveniles. El principal factor de riesgo fue la edad. La definición de lesión según tiempo perdido fue insuficiente para el tamizaje de lesiones por sobreuso, pesquisando una minoría de los jugadores afectados.

Objective To determine the prevalence of overuse injuries in the senior and junior teams of the Chilean and Uruguayan rugby national teams. Secondary objectives: 1 . Describe the impact on performance, risk factors and differences between senior players and junior players; 2 . Evaluate the time lost injury definition for studies in overuse injuries. received

September 30, 2016 accepted

December 2, 2016
DOI http://dx.doi.org/ $10.1055 / \mathrm{s}-0037-1599198$ ISSN $0716-4548$.
Copyright $\odot 2017$ by Thieme-Revinter

Publicações Ltda, Rio de Janeiro, Brazil
License terms

(c) (i) $\ominus$ (\$) 


\author{
Keywords \\ - rugby \\ - overuse injuries \\ - rugby injuries
}

Method Study group: senior and junior national rugby teams of Chile and Uruguay (106 players). The "Oslo Sports Trauma Research Centre Overuse Injury Questionnaire" was used for the registration and evaluation of overuse injuries. An overuse injury was defined as all joint problems of non-traumatic origin, which occurred up to 7 days prior to the interview. Age, weight, height, playing position and training volume were recorded for the analysis of risk factors.

Results The prevalence of overuse injuries was 31\%. Only 6\% required more than 24 hours of rest from sports. The main risk factor was age (OR $1.12 \mathrm{Cl} 1.017$ to 1.24 ), increasing by $12 \%$ for every year of age. A higher prevalence in senior players was noted, however, juniors had a higher severity score than adults (23.2 versus $15.8 p=0.03$ ). Conclusions The prevalence of overuse injuries in this group was high. The impact on performance was mild in most players, being higher in juniors. The main risk factor was age. The time lost injury definition was insufficient for the screening of overuse injuries, only diagnosing a minority of the affected players.

\section{Introducción}

En la actualidad, las lesiones por sobreuso han recibido poca atención en la literatura internacional. Los datos válidos y confiables son escasos y los estudios en prevención casi inexistentes. Esto se debe, probablemente, a las dificultades en la metodología del estudio de las lesiones por sobreuso. Las lesiones por sobreuso presentan una serie de características particulares que las hacen difíciles de estudiar con métodos tradicionales de investigación en lesiones deportivas.

La metodología actual para la investigación en lesiones deportivas basa sus definiciones en el consenso del año 2006 de la Fédération Internationale de Football Association Medical Assessment and Research Centre (F-MARC). ${ }^{1}$ En su consenso para la vigilancia de lesiones en el fútbol, se definió una lesión como cualquier molestia física, independiente de que requiera atención médica o reposo deportivo. Es una definición amplia, que no limita la inclusión de lesiones debido a la disponibilidad y el acceso del deportista a una atención médica y tampoco condiciona la inclusión de lesiones a un tiempo de reposo mínimo por la lesión.

En el 2007, se publicó el consenso para la vigilancia de lesiones del rugby, creado por un grupo de trabajo de World Rugby (antigua International Rugby Board). ${ }^{2}$ Este consenso, basado en el consenso de la F-MARC, puso mayor énfasis en las lesiones que ocasionen un reposo deportivo mayor a 24horas. Esto tuvo como consecuencia, una menor inclusión de lesiones que la usada en la primera definición propuesta en el consenso de la F-MARC, debido a que dejó de incluir lesiones que no tuviesen un impacto en el tiempo perdido por lesión. Desde esa fecha en adelante, la definición de lesión por tiempo perdido ha sido la más popular en todos los trabajos epidemiológicos de lesiones deportivas en los distintos deportes. La definición de lesión, como solo cualquiera que impida el retorno deportivo antes de 24 horas. luego de ocurrido el evento, tiene la ventaja de que es independiente del acceso a la atención médica, permite la comparación de datos a distintos niveles deportivos, es objetiva y traduce un impacto medible como consecuencia de la lesión, tanto en el deportista como en su equipo. La limitación es, que en el caso de las lesiones por sobreuso, probablemente solo incluya las lesiones de mayor gravedad. Esto debido a que la mayoría de los deportistas continúan entrenando y compitiendo a pesar de la presencia de una lesión por sobreuso, adaptando los niveles de carga y los ejercicios realizados, muchas veces, sin ni siquiera solicitar una consulta médica. Por lo tanto, solo en el menor de los casos producen un reposo deportivo efectivo. ${ }^{3}$

Una vez que la lesión es incluida según la definición anterior, el consenso de World Rugby define la categorización de una lesión por sobreuso a aquella lesión en donde no se logra identificar un evento traumático desencadenante. Además, algunos autores han agregado a esta definición el concepto de inicio gradual de los síntomas. ${ }^{2}$ En algunas ocasiones puede ser fácil diferenciar entre una lesión traumática de una no traumática, pero otras veces es muy difícil. Eso se debe a que existen lesiones que inician sus síntomas de forma brusca, pero son la consecuencia de un proceso fisiopatológico más largo, como por ejemplo en el caso de las fracturas por estrés.

Por lo anteriormente explicado es que, como primera etapa, se hace necesario conocer la magnitud del problema en el rugby y se plantean las siguientes preguntas: 1 . ¿Existen las lesiones por sobreuso en el rugby? 2. ¿Cuál es el impacto de las lesiones por sobreuso? 3. ¿Están las lesiones por sobreuso sub-diagnosticadas en el rugby?

El objetivo principal de este estudio fue determinar la prevalencia de lesiones por sobreuso en el plantel adulto y juvenil (menores de 20 años) de la selección chilena y la selección uruguaya de rugby. Como objetivos secundarios estuvieron: 1. Describir el impacto en el rendimiento en los jugadores; 2 . Determinar los factores de riesgo y comparar las diferencias entre los planteles adultos y juveniles; 3 . Evaluar la definición de lesión según tiempo perdido para lesiones por sobreuso en este grupo. 


\section{Metodología}

\section{Grupo de estudio}

En un trabajo colaborativo entre la Federación de Rugby de Chile y la Unión de Rugby Uruguaya, se definió un grupo de estudio compuesto por los planteles de las selecciones de rugby adulta de Chile (27 jugadores) y Uruguay (26 jugadores) previo al campeonato sudamericano adulto de rugby 2013 y los planteles de las selecciones de rugby juveniles de Chile (26 jugadores) y Uruguay (27 jugadores), ambos previo al campeonato mundial juvenil de rugby 2013. Se incluyeron el total de jugadores seleccionados para los planteles descritos. Todos los jugadores incluidos fueron inscritos en la nómina definitiva de los torneos respectivos y se encontraban disponibles para jugar al momento del registro. El total de jugadores incluidos fue de 106 .

\section{Instrumento de evaluación}

Se utilizó el Oslo Sports Trauma Research Centre (OSTRC) Overuse Injury Questionnaire validado en el año 2012 para el tamizaje y la evaluación inicial de las lesiones por sobreuso. ${ }^{4}$ Como criterio de inclusión, este cuestionario reemplaza el término "lesión" por "problema," definiendo "problema" como dolor, rigidez, aumento de volumen, bloqueos, o inestabilidad de origen no traumático.

El cuestionario fue aplicado mediante una entrevista personal con cada jugador por el médico del equipo y fueron considerados para la inclusión de lesión por sobreuso todos los "problemas" articulares de origen no traumático, ocurridos hasta los 7 días previos a la entrevista. Luego de identificar un problema articular, el médico del equipo fue el responsable de determinar, mediante la anamnesis dirigida, si el problema articular tuvo un origen no traumático para ser incluida en el estudio como un caso.

A cada caso, se aplicó el cuestionario de 4 preguntas para cada problema articular identificado, para evaluar el impacto en el rendimiento de cada lesión en el jugador (-Fig. 1). Cada pregunta tuvo un puntaje de 0 a 25 puntos divididos en partes iguales por cada repuesta, dando una escala general de 0 a 100 puntos por las 4 preguntas sumadas, siendo 0 puntos sin impacto y 100 puntos el mayor impacto en el rendimiento deportivo.

Para el análisis descriptivo se agruparon las lesiones en 7 regiones: rodilla; hombro; mano, muñeca y codo; tobillo y pie; columna lumbar; columna cervical; cadera y pelvis.

\section{Factores de riesgo}

Para el análisis de factores de riesgo se registró en todos los jugadores la edad, peso, talla y posición de juego. Además se registró el volumen de entrenamiento evaluando 3 factores: 1. La cantidad de meses seguidos entrenando desde la última pausa de al menos 3 semanas; 2 . Número de sesiones de entrenamiento por semana durante los últimos 2 meses; 3. Tiempo promedio de duración de cada sesión de entrenamiento durante los últimos 2 meses; 4 . Cantidad total estimada de horas de entrenamiento en los últimos 2 meses (calculado desde los puntos 2 y 3 ).

\author{
Question 1 \\ Hove you had any difficulties participating in normal training \\ and competition due to knee problems during the past week? \\ Full participation without knee problems \\ Full participation, but with knee problems \\ Reduced participation due to knee problems \\ Cannot participate due to knee problems \\ Question 2 \\ To what extent have you reduced you training volume due to \\ knee problems during the past week? \\ No reduction \\ To a minor extent \\ To a moderate extent \\ To a major extent \\ Cannot participate at all \\ Question 3 \\ To what extent have knee problems affected your performance \\ during the past week? \\ No effect \\ To a minor extent \\ To a moderate extent \\ To a major extent \\ Cannot participate at all \\ Question 4 \\ To what extent have you experienced knee pain related to your \\ sport during the post week?

\section{No pain} \\ Mild pain \\ Moderate pain \\ Severe pain
}

Fig. 1 Ejemplo de las 4 preguntas del cuestionario OSTRC Overuse Injury Questionnaire para problemas en la rodilla.

\section{Evaluación de la definición según tiempo perdido y conducta de los jugadores}

Para cada caso incluido, se evaluó si requirió un tiempo de reposo deportivo mayor a 24 hrs debido a la lesión, lo que permitió comparar la prevalencia de lesiones por sobreuso según la definición de OSTRC y World Rugby. Además, en cada caso incluido, se registró si el jugador solicitó una evaluación médica y si tenía un diagnóstico específico al momento de la entrevista.

\section{Análisis estadístico}

Se realizó un análisis estadístico descriptivo y analítico. Se realizaron regresiones logísticas multivariadas para buscar asociación entre las variables analizadas y la presencia de una lesión. Se realizó un t-test para la comparación de medias y proporciones entre los grupos adultos y juveniles. Se 
Tabla 1 Descripción de puntaje de gravedad de las lesiones por sobreuso

\begin{tabular}{|l|l|l|l|l|l|}
\hline $\mathbf{N}^{\circ}$ & Min & Max & p50 & Media & DS \\
\hline 40 & 6 & 53 & 14 & 17,83 & 11,9 \\
\hline
\end{tabular}

Abreviaciones: DS, desviación estandar; Max, maximo; Min, minimo.

consideraron resultados significativos para un $p<0,05$. Para todo el análisis se utilizó el programa Stata 11.2.

\section{Resultados}

La prevalencia de lesiones por sobreuso en el grupo de estudio fue de un $31 \%$, con un total de 40 lesiones en 33 jugadores. De los jugadores con lesión, solo el $6 \%{ }^{2}$ requirió más de 24 hrs de reposo deportivo. El $100 \%$ de las lesiones definidas como "problemas" fueron identificadas solo con el síntoma de dolor, con un puntaje EVA promedio de 4 (rango: 2-7). El 52\% (17) de los jugadores lesionados buscó asistencia médica previo al estudio y el 48\% (15) tenía diagnóstico específico.

El factor edad fue el único factor de riesgo identificado de forma significativa para presentar una lesión, con un OR de 1,12 ( $p<0,05$ IC $1,017-1,24)$. Esto determinó un aumento del riesgo de presentar una lesión en un $12 \%$ por cada año de edad cumplido. Ningún otro factor analizado se asoció a un aumento del riesgo de presentar una lesión por sobreuso.

La descripción del puntaje de gravedad de las lesiones identificadas se puede observar en la Tabla 1.

Al comparar la prevalencia de lesiones entre los equipos adultos y juveniles se observa una mayor prevalencia significativa en el grupo de seleccionados adultos (-Fig. 2). El análisis entre los puntajes de gravedad de ambos grupos se puede observar en la - Fig. 3. Los juveniles presentaron un puntaje de gravedad significativamente mayor que los adultos (23,2 versus $15,8 p=0,03$, rango: 6 - 53). El $20 \%$ de las lesiones presentó un puntaje mayor a 25 puntos.

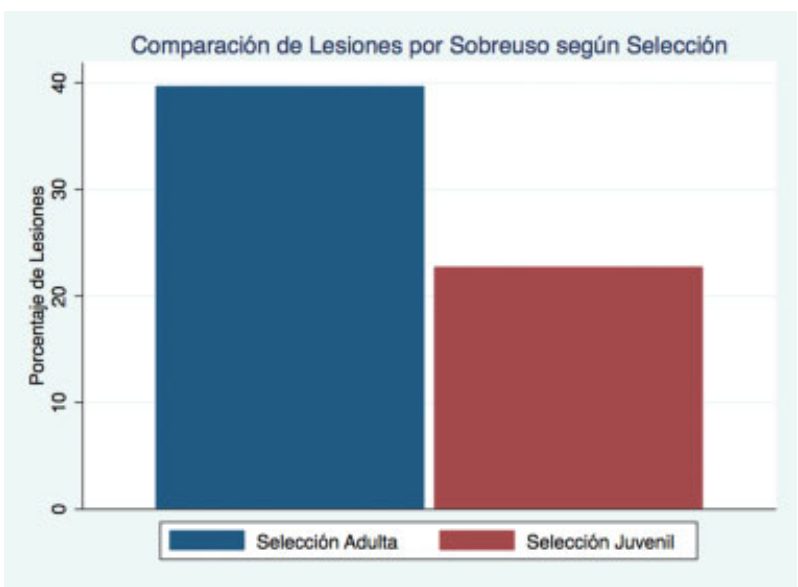

Fig. 2 Comparación de prevalencia de lesiones por sobreuso entre selecciones juveniles y selecciones adultas

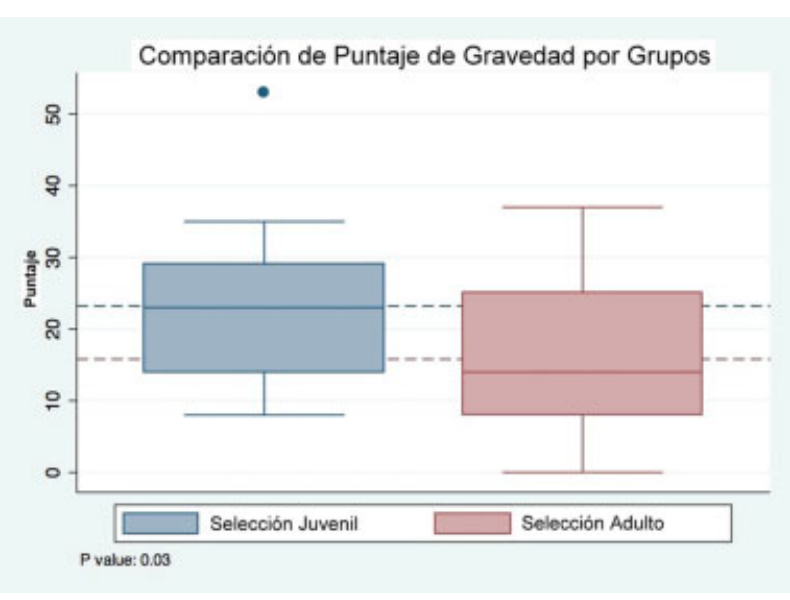

Fig. 3 Comparación de puntaje de gravedad de lesiones por sobreuso entre selecciones juveniles y selecciones adultas.

\section{Discusión}

El registro de las lesiones por sobreuso presenta características que dificultan su estudio: los deportistas en general, no detienen su práctica deportiva al inicio del dolor. La respuesta habitual de los deportistas al dolor ha sido descrita previamente como un patrón de conducta que se repite de forma constante. ${ }^{2,3,5}$ Primero se dan cuenta del dolor. Luego el dolor aumenta con las mismas actividades, por lo que deciden bajar el nivel de carga y/o modificar los ejercicios o actividades desencadenantes. Luego, el dolor aumenta hasta llegar a la intensidad necesaria según el umbral individual, para que el deportista detenga su actividad de forma completa. Cuando el dolor disminuye nuevamente por debajo de ese umbral, intenta volver a sus actividades, hasta que el dolor nuevamente lo obliga a realizar reposo deportivo. Luego de múltiples intentos por volver, consideran mayor reposo y tratamiento médico asistido para lograr el retorno deportivo definitivo.

En el 2013, se publicó la primera meta-análisis de lesiones en jugadores de rugby adultos profesionales. Esta investigación incluyó 15 estudios desde el año 2005 en adelante, en donde 12 incluyeron como lesión a aquellas que hayan provocado tiempo perdido mayor a $24 \mathrm{hrs}$. En todo el metaanálisis, no se mencionó la presencia de lesiones por sobreuso. $^{6}$ Esto, a pesar de que, en el formulario oficial para el registro de lesiones en el rugby publicado junto al consenso del 2007, existe un punto en dónde se debe clasificar la lesión registrada entre traumática o por sobreuso. ${ }^{2}$ También, en un estudio epidemiológico de lesiones en el rugby chileno, con un seguimiento de 2 años a la selección juvenil de chile, que incluyó un total de 94 jugadores y 70 lesiones analizadas, no se logró incluir ninguna lesión por sobreuso que cumpliera con el criterio de la definición utilizada. ${ }^{7}$

Con la metodología propuesta bajo la definición de OSTRC en este trabajo para el tamizaje de lesiones por sobreuso, se logró identificar un número de lesiones 20 veces mayor que con la metodología tradicional propuesta por World Rugby (40 versus 2). Lo importante de esto es 
que existe evidencia de que la presencia de dolor y su experiencia en los humanos, está asociado a alteraciones en la atención, memoria de trabajo, flexibilidad mental, capacidad de resolver problemas y velocidad de procesamiento de la información. Además, se asocia a un aumento de la ansiedad, el miedo y la ira. ${ }^{8}$ Si se logra identificar de mejor manera este tipo de lesiones se podrá optimizar el rendimiento de los deportistas desde lo mental a lo físico.

Ambos métodos son difíciles de comparar. Esta nueva metodología incluye un gran número de problemas, aunque el único síntoma sea dolor leve. Por lo tanto, es esperable que esto incluya un mayor número de lesiones, aunque el impacto en el deportista sea leve. Creemos que si el objetivo es el tamizaje, es preferible detectar un mayor número potencial de lesiones, para luego realizar el diagnóstico específico y no subdiagnosticar las lesiones. Dentro de sus ventajas para el tamizaje de las lesiones por sobreuso están el uso de prevalencia en vez de incidencia y la utilización de un puntaje de gravedad que refleja una autoevaluación del deportista con respecto a su dolor y al impacto que este tiene en su rendimiento. Utilizar una clasificación de gravedad basada en la cantidad de días perdidos por lesión es una medida objetiva útil, pero puede no reflejar todo el impacto de las lesiones por sobreuso.

Dentro de las limitaciones de la metodología y del estudio, tenemos las siguientes: que la información obtenida depende de la honestidad de los deportistas; la herramienta aún no ha sido validada al español; puede existir un sesgo del entrevistador al interpretar la información obtenida; la muestra de este estudio es pequeña y no representa una prevalencia promedio en el tiempo. Por último tenemos que algunas de las lesiones registradas con esta herramienta de tamizaje, pudiesen corresponder a un dolor relacionado con la actividad deportiva, (como, por ejemplo, dolor muscular de inicio tardío), sobrediagnosticando las lesiones por sobreuso, ya que no incluye el diagnóstico específico. Sin embargo, de todas formas, permite identificar problemas articulares que pudiesen tener un impacto negativo sobre el deportista en el momento de la evaluación. Proponemos para el futuro, evaluar la sensibilidad y especificidad de la herramienta OSTRC para el tamizaje de lesiones por sobreuso específicamente en el rugby.

\section{Conclusiones}

A diferencia de la evidencia disponible, la prevalencia de dolor que pudiese corresponder a lesiones por sobreuso fue alta en los seleccionados de rugby adulto y juvenil de Chile y Uruguay, inmediatamente antes del torneo más importante de cada equipo ese año. El impacto en el rendimiento percibido por la mayoría de los deportistas fue leve, siendo significativamente mayor en los juveniles. El principal factor de riesgo identificado fue la edad, aumentando el riesgo de forma significativa por cada año de edad cumplido. Observamos que la definición según tiempo perdido usada en la metodología tradicional fue insuficiente para este tipo de lesiones, ya que solo logró pesquisar una minoría de los jugadores afectados. Por esta razón, se recomienda considerar metodología específica para el estudio de las lesiones por sobreuso, considerando que el tamizaje de estas lesiones sigue siendo un desafío para la metodología científica.

\section{Referencias}

1 Clarsen B, Myklebust G, Bahr R. Development and validation of a new method for the registration of overuse injuries in sports injury epidemiology: the Oslo Sports Trauma Research Centre (OSTRC) overuse injury questionnaire. Br J Sports Med 2013; 47(08):495-502

2 Bahr R. No injuries, but plenty of pain? On the methodology for recording overuse symptoms in sports. Br J Sports Med 2009; 43(13):966-972

3 Fuller CW, Ekstrand J, Junge A, et al. Consensus statement on injury definitions and data collection procedures in studies of football (soccer) injuries. Br J Sports Med 2006;40(03):193-201

4 Fuller CW, Molloy MG, Bagate C, et al. Consensus statement on injury definitions and data collection procedures for studies of injuries in rugby union. Br J Sports Med 2007;41(05):328-331

5 Clarsen B, Bahr R, Heymans MW, et al. The prevalence and impact of overuse injuries in five Norwegian sports: Application of a new surveillance method. Scand J Med Sci Sports 2015;25(03): 323-330

6 Williams S, Trewartha G, Kemp S, Stokes K. A meta-analysis of injuries in senior men's professional Rugby Union. Sports Med 2013;43(10):1043-1055

7 Marsalli M, Soza F, García N, Morán N. Análisis epidemiológico de lesiones de la selección chilena de rugby menores de 20 años. Rev Chil Ortop Traumatol 2013;54(03):105-111

8 Simons LE, Elman I, Borsook D. Psychological processing in chronic pain: a neural systems approach. Neurosci Biobehav Rev 2014;39(02):61-78 